\title{
Energy spectra of X-ray quasi-periodic oscillations in accreting black hole binaries
}

\author{
P. T. Życki, ${ }^{1}$ M. A. Sobolewska ${ }^{2}$ and A. Niedźwiecki ${ }^{3}$ \\ ${ }^{2}$ Nicolaus Copernicus Astronomical Center, Bartycka 18, 00-716 Warsaw, Poland \\ ${ }^{2}$ Department of Physics, University of Durham, South Road, Durham DH1 3LE, UK \\ ${ }^{3}$ Eódź University, Department of Physics, Pomorska 149/153, 90-236 Lódź, Poland \\ email: ptz@camk.edu.pl
}

\begin{abstract}
We investigate the energy dependencies of X-ray quasi-periodic oscillations in black hole X-ray binaries. We analyze RXTE data on both the low- and high-frequency QPO. We construct the low- $f$ QPO energy spectra, and demonstrate that they do not contain the thermal disk component, even though the latter is present in the time averaged spectra. The disk thus does not seem to participate in the oscillations. Moreover the QPO spectra are harder than the time averaged spectra when the latter are soft, which can be modeled as a result of modulations occurring in the hot plasma. The QPO spectra are softer than the time averaged spectra when the latter are hard. The absence of the disk component in the QPO spectra is true also for the high-frequency (hecto-Hz) QPO observed in black hole binaries. We compute the QPO spectra expected from the model of disk resonances.
\end{abstract}

Keywords. Accretion, accretion disks - relativity - X-rays: binaries

\section{Introduction}

Quasi-periodic components of X-ray variability are often seen in power density spectra (PDS) from accreting compact objects, signifying the existence of "clocks" operating on a specific time scale, among the broad-band variability. The nature of these periodic processes is unknown, but they are usually associated with various modes of oscillations of standard accretion disks present in those systems.

Energy dependencies of the QPO can be an important clue to the origin of these features, similarly to analyzes of time averaged energy spectra, which provide information about physical processes of generation of X-rays. It was noted quite early on into the study of QPO that their r.m.s. variability seems to increase with energy (review in van der Klis 2006). Here we report on more detailed investigations of QPO energy dependencies in black hole X-ray binaries. Firstly, we construct observed low- $f(1-10 \mathrm{~Hz})$ QPO energy spectra in the 2-20 keV range using RXTE data. Secondly, we construct models of spectral variability of inverse-Compton emission, producing a given type of QPO spectra. We also analyze data on high- $f$ (hecto-Hz) QPO and construct models of energy spectra of these QPO in the model of disk resonances (Abramowicz \& Kluźniak 2001).

\section{Low-frequency QPO}

\subsection{Observations}

We analyzed observations of four sources: GRS 1915+105, XTE 1550-564, 4U1630-47 and XTE $1859+226$ performed by RXTE in observing modes providing good energy and timing resolution. Details of data reduction and analysis procedures are given in Sobolewska 

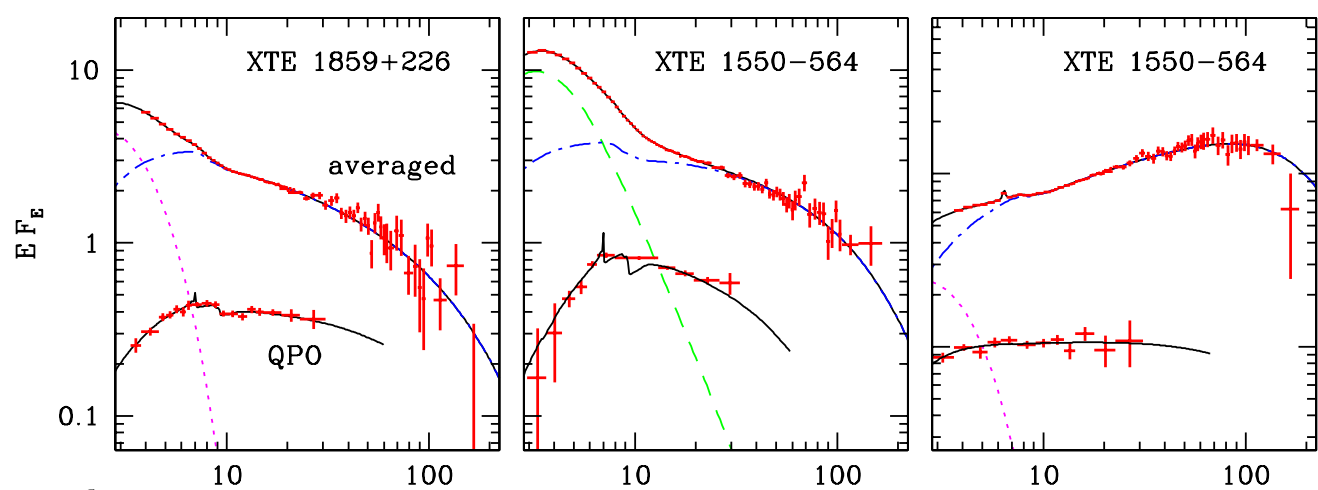

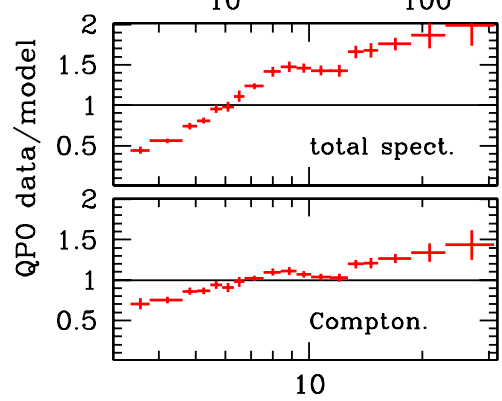

Energy $[\mathrm{keV}]$

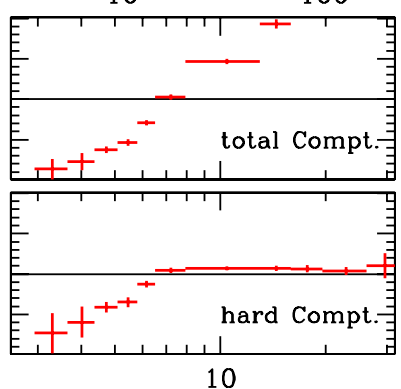

Energy $[\mathrm{keV}]$

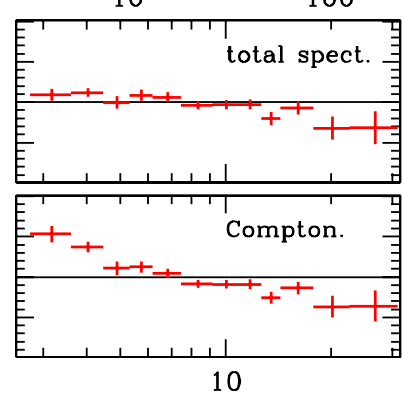

Energy [keV]

Figure 1. Comparison of time averaged spectra and the QPO spectra (upper panels). Lower panels show ratios of the QPO spectra to various components from the time averaged spectra.

\& Życki (2006). Firstly, we construct time-averaged energy spectra in the $3-200 \mathrm{keV}$ energy range using PCA and HEXTE data. These are fit with a model containing two or three continuum components (disk black body, comptonization) and the reprocessed component (Fe $\mathrm{K} \alpha$ line and the Compton reflected continuum). Then we construct the QPO energy spectra: we compute PDS in each energy channel, then fit it with a model consisting of a broad band continuum (broken power law or a number of Lorentzians) and one or two narrow Lorentzian peaks representing the QPO, possibly with a harmonic. The QPO Lorentzians are then integrated over frequency. This, expressed as variance (rather than the usual r.m.s./mean) is the QPO energy spectrum. The meaning of such a function is that it would correspond to energy spectrum of a variable component, if it was a separate spectral component which would be responsible to the QPO.

The results are presented in Fig 1 . We show both the time averaged spectra and the QPO spectra. We also show the ratios of QPO spectra to the time averaged models. The main result is the lack of the soft disk component in the QPO spectra. Presence of this is excluded in all analyzed spectra at high confidence level. This can be seen for example in the spectra of XTE $1859+226$, where the QPO spectra are much closer to the Comptonized component only than to total time averaged spectra. Secondly, we analyzed the slope of the QPO spectra relative to the time averaged spectra. In soft spectral state the QPO spectra are clearly harder than the time averaged spectra (left), while the opposite is true for the hard state (right). During the two observations of 4U1630-47 the time averaged spectra are intermediate in slope between values typical for the soft and hard states $(\Gamma \approx 2)$. We find that the QPO spectra are similar to the time averaged spectra in these two observations. 

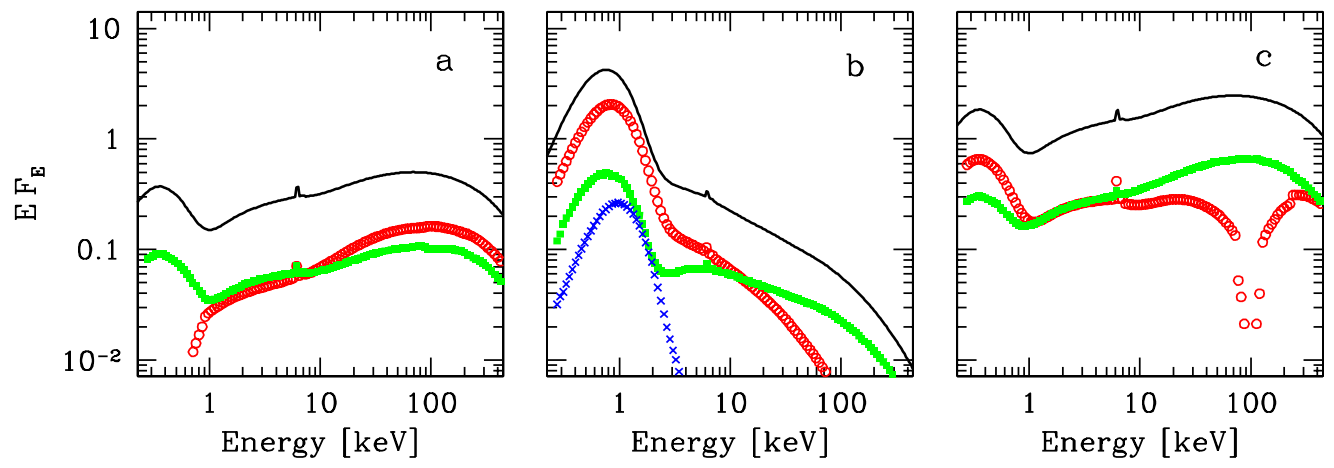

Figure 2. Model predictions for the QPO energy spectra (red circles) in various scenarios for modulations: a) heating; b) cooling; c) covering factor of cold matter. Green squares are the broad band noise spectra, blue stars: QPO harmonic, solid line shows time averaged spectra.

\subsection{Models}

In an attempt to understand the results presented in the previous Section, we performed simulations of spectral variability of inverse-Compton component. Such spectra are described by three main parameters: the plasma heating rate, $l_{\mathrm{h}}$, the flux of cooling soft photons, $l_{\mathrm{s}}$, and the temperature of the input soft photons, $T_{0}$. Irrespectively on the physical mechanism of QPO production, it is one of the three parameters that has to be modulated if the observed QPO corresponds to modulation of intrinsic luminosity (other possibility is a periodic modulation of geometry affecting, e.g., relativistic effect influencing the observed luminosity; see below).

The slope of the time averaged Comptonized spectrum depends (almost) only on the ratio $l_{\mathrm{h}} / l_{\mathrm{s}}$, however the mode of spectral variability depends on which parameter is modulated. Details of this investigation are given in Życki \& Sobolewska (2005). To summarize it: modulation of the heating rate produces variability spectrum with largest amplitude of variability at high energies, which means that the variability spectrum is harder than the time averaged spectrum. Modulation of the cooling rate or the soft photons temperature produces variability spectrum softer than the time averaged spectrum. We considered also modulation of the amplitude of the reprocessed component, which was assumed to relate the cooling and heating rate (see, e.g., Poutanen \& Fabian 1999). In this case we also find that the QPO are softer than the time averaged spectra, but, additionally, a very strong $\mathrm{Fe} \mathrm{K} \alpha$ line is seen in the QPO spectrum.

Summarizing, properties of low- $f$ QPO spectra in the soft state strongly suggest that these QPO are generated as a result of modulations in the hot plasma rather than in the standard cold accretion disk. This is contrary to most theoretical ideas where oscillations of the cold disk are thought to be the primary driver. However, the physical mechanism does seem to require both accreting plasma phases to work, since these QPO are associated with changes of the geometry of the system. It is also interesting to note, that long-time scale variability of $\mathrm{Cyg} \mathrm{X}-1$ in the soft state also seems to be driven by instabilities in the hot Comptonizing plasma (Zdziarski et al. 2002).

\section{High-frequency QPO}

Data on high-frequency QPO in black hole X-ray binaries are of much poorer quality than data for the low- $f$ QPO. It is nevertheless clear that they share at least one characteristics with their low- $f$ counterparts, namely the soft disk component is absent 

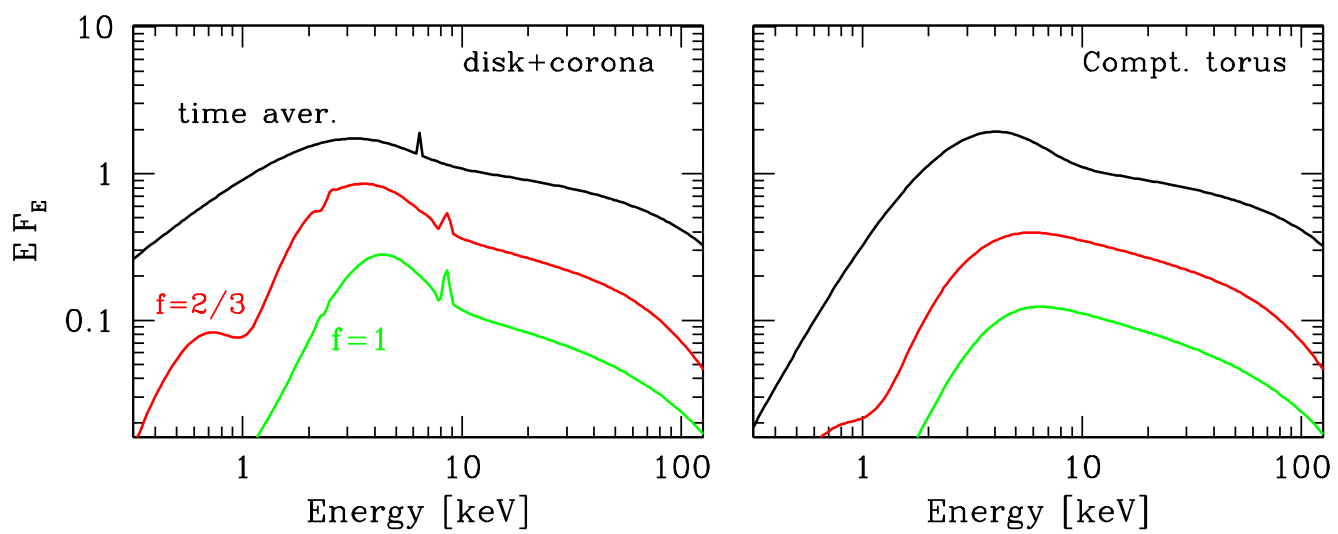

Figure 3. QPO spectra from the model of epicyclic oscillations, with relativistic effects modulating the X-ray flux, for $a=0.998$ and inclination $\mu_{\mathrm{obs}}=0.3$. Left: geometry of accretion disk with an active corona produces strong disk component in the QPO spectra. Right: hot oscillating torus produces QPO spectrum resembling a comptonization component.

in the QPO spectra. More detailed energy dependencies (i.e., the spectral slope) are not possible to determine with current data.

The fact that pairs of QPO with 3:2 frequency ratio are sometimes observed stimulated development of models invoking non-linear resonances between various modes of oscillations of accretion disks. In particular, Abramowicz \& Kluźnika (2001) postulate that the relevant modes are the vertical and radial epicyclic motions. Importantly, they suggest also that the X-ray flux modulation occurs due to changes of geometry and, consequently, changes in the strength of the relativistic effects (Doppler and gravitational shifts; light bending), with the primary emission being constant. This is thus the only current model making definite predictions for the more detailed properties of the QPO, for example, their energy spectra. We used the code of Życki \& Niedźwiecki (2005) for photon transfer in Kerr metric and modified it to implement the source motion corresponding to disk epicyclic oscillations (details are in Życki et al., in preparation).

We have performed computations of the relativistic effects expected from such disks at radii corresponding to $3: 2$ resonance between the oscillation frequencies, and we have computed energy spectra of QPO expected in this model. They are presented in Fig. 3 in two variants of geometry: (1) accretion disk extending to the last stable orbit with an active X-ray corona, and (2) a truncated accretion disk with inner hot Comptonizing flow.

In the first scenario the QPO spectrum contains a strong disk component, since the disk emission is modified by the relativistic effects. In the second scenario the disk is, by assumption, not present at the QPO radius and, as a consequence, only the Comptonized component is modulated. The Fe $\mathrm{K} \alpha$ line is also assumed to be produced at the QPO radius (in the accretion disk with a corona geometry) and it does appear in the QPO spectrum, at positions corresponding to maximum redshift expected from the oscillatory motion.

\section{Acknowledgements}

This work was partially supported by grant 2P03D01225 from Polish Ministry of Science (MNIiI). 


\section{References}

Abramowicz M. A. \& Kluźniak W. 2001, A\&A, 374, L19

Poutanen J. \& Fabian A. C. 1999, MNRAS, 306, L31

Sobolewska M. A. \& Życki P. T. 2006, MNRAS, 370, 405

van der Klis M. 2006, in: W. H. G. Lewin \& M. van der Klis (eds.), Compact Stellar X-ray Sources (Cambridge University Press: Cambridge), p. 39

Zdziarski A. A., Poutanen J., Paciesas W. S., Wen L. 2002, ApJ, 578, 357

Życki P. T. \& Niedźwiecki A. 2005, MNRAS, 359, 308

Życki P. T. \& Sobolewska M. A. 2005, MNRAS, 364, 891

SuZY Collin: According to your model, should QPOs be visible in AGN?

PIOTR ŻYCKI: Formulation of the model I described is too general to resolve the question whether QPOs should appear and be visible in AGN. I understand there are mainly observational issues - AGN data are only marginally good to search for QPOs. There are also claims that QPO detectability depends on the inclination angle but again this is beyond the scope of the present model.

Mitchell Begelman: How much information can you extract about properties of the Comptonizing cloud from the energy dependency of the QPO signal?

PIOTR ŻYCKI: Information about optical depth or temperature are better constrained from time averages spectra, because statistics is much better. We deal mostly with the question of what actually oscillates - whether it is hot plasma or whether it is a cold disc that oscillates. This kind of issues we address. If the data are very good, we could in principle distinguish whether it is optical depth or temperature changes that are causing the oscillations.

It seems that low-frequency oscillations are produced by processes in a corona rather than a cold accretion disc. A mechanism that produces oscillations in a hot corona is implied by our model. 


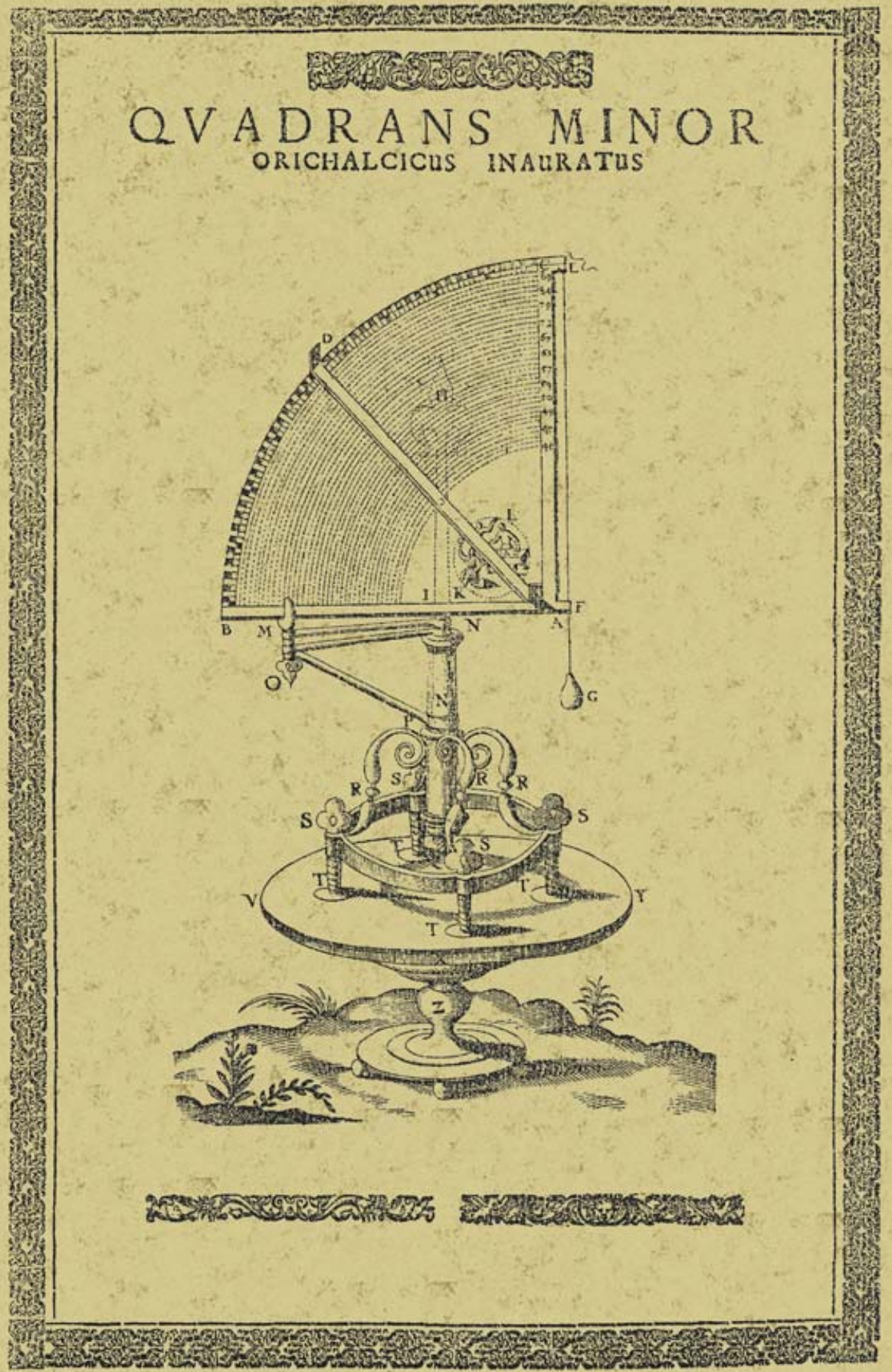

